\title{
Corticosteroid Injections After Rotator Cuff Repair Improve Function, Reduce Pain, and Are Safe: A Systematic Review
}

\author{
Richard N. Puzzitiello, M.D., Bhavik H. Patel, M.D., Ophelie Lavoie-Gagne, M.D., \\ Yining Lu, M.D., Benedict U. Nwachukwu, M.D., M.B.A., Brian Forsythe, M.D., and \\ Matthew J. Salzler, M.D.
}

\begin{abstract}
Purpose: To review the literature on postoperative corticosteroid injections (CSIs) following primary rotator cuff repair (RCR) to evaluate efficacy and adverse effects. Methods: A systematic review of the MEDLINE, EMBASE, and Cochrane databases were performed to identify all studies published within the last 15 years, which reported on outcomes of postoperative CSIs following RCR. Studies including patients who received only preoperative CSIs and revision RCRs were excluded. Included studies were evaluated for study methodology, patient demographics, outcome measures, physical examination parameters, results of imaging studies, and adverse effects or clinical complications. Results: Seven studies comprising 5,528 patients satisfied inclusion criteria. Among included patients, 54.8\% were female and mean age range from $52.3 \pm 13.0$ to $62.7 \pm 6.6$ years. Only 1 included investigation was a Level I study. Overall, 4 of 5 studies reported significant improvements in pain and outcome scores (Constant score, American Shoulder and Elbow Surgeons score) compared with controls. Across all studies, the majority of these effects were statistically significant at 3 months postoperatively but not beyond this time point. Five of the 6 included investigations reported no increased rate of retears after postoperative CSIs. One study did find an increase in retear in patients receiving postoperative CSIs but was unable to determine whether these retears were present before the patient received the CSI. Another investigation reported an increased rate of infection only if the CSI was administered in the first postoperative month. Conclusions: Postoperative CSIs may improve pain and function for up to 3 months following primary RCR but not at later follow-up time points. CSIs should be administered only after the first postoperative month to minimize the potential risk for adverse events. Level of Evidence: Systematic review of level I-IV studies.
\end{abstract}

C orticosteroid injections (CSIs) have become commonplace in the realm of orthopaedic surgery ${ }^{1}$ and are now used in the treatment of several prevalent conditions, including osteoarthritis, ${ }^{1}$ adhesive capsulitis of the shoulder, ${ }^{2}$ and various tendon pathologies. ${ }^{3,4} \mathrm{~A}$ number of options for injections exist, including hydrocortisone, betamethasone, methylprednisolone, triamcinolone, and dexamethasone. ${ }^{5}$ These drugs exert a complex anti-inflammatory effect on injected tissues

From the Department of Orthopaedics, Tufts Medical Center; Boston, Massachusetts (R.N.P., M.J.S.); Department of Orthopaedic Surgery, University of Illinois, Chicago, Illinois (B.H.P.); Harvard Combined Orthopaedic Residency Program, Boston, Massachusetts (O.L.-G.); Department of Orthopaedic Surgery, Mayo Clinic, Rochester, Minnesota (Y.L.); Department of Orthopedic Surgery, Hospital for Special Surgery; New York, New York (B.U.N.); and Midwest Orthopaedics at Rush, Rush University Medical Center; Chicago, Illinois (B.F.), U.S.A.

The authors report the following potential conflicts of interest or sources of funding: B.F. reports personal fees from Elsevier, Arthrex, Jace Medical, and Stryker; grants from Smith $\theta$ Nephew and Ossur, outside the submitted work. M.J.S. reports editorial or governing board for Arthroscopy and Journal of Bone and Joint Surgery, American, and board member/committee appointments for a society: AAOS and American Orthopaedic Society for Sports by counteracting proinflammatory cytokines, downregulating immune function, and dampening vascular responses. ${ }^{6}$ The net result is often a reduction in pain and stiffness, with substantial relief conferred to the patient in the short- to intermediate-term. ${ }^{7}$

One application of CSIs that has garnered considerable interest in recent years is their use in rotator cuff pathology. In 2 recent meta-analyses, Lin et al. ${ }^{8}$ and Mohamadi et al. ${ }^{3}$ found that corticosteroids had limited

Medicine. Full ICMJE author disclosure forms are available for this article online, as supplementary material.

Received February 6, 2020; accepted October 11, 2021.

Address correspondence to Bhavik H. Patel, M.D., Midwest Orthopaedics at Rush, Rush University Medical Center, 1611 W. Harrison St., Suite 300, Chicago,IL60621.E-mail: bhavik91@gmail.com or forsythe.research@ rushortho.com

(C) 2021 Published by Elsevier Inc. on behalf of the Arthroscopy Association of North America. This is an open access article under the CC BY-NC-ND license (http://creativecommons.org/licenses/by-nc-nd/4.0/).

2666-061X/20145

https://doi.org/10.1016/j.asmr.2021.10.010 
and transient utility in reducing pain and increasing function for patients with rotator cuff tendinopathy. Moreover, several studies have recently established significant relationships between preoperative CSIs and postoperative complications following rotator cuff repair (RCR) including retear, the need for revision surgery, and infection. ${ }^{9-14}$ These complications associated with preoperative CSIs also may apply to postoperative CSIs after RCR. Basic science investigations have additionally reported that local administration of glucocorticoids can be deleterious to tendon health and physiology, inducing changes such as reduced cellular viability/proliferation, diminished mechanical strength, increased necrosis, and disorganization of the extracellular matrix. ${ }^{15}$ Given this growing body of evidence challenging the role of CSIs in rotator cuff pathology, this topic remains actively debated within the orthopaedic community.

In the midst of this discussion, several groups also have investigated the use of CSIs in the postoperative setting. ${ }^{9,16-21}$ Pain and stiffness are among the most common complications following $\mathrm{RCR}^{22,23}$ and there may be a meaningful role for steroid injections in management. However, this application of CSIs is poorly understood, and neither the efficacy nor safety of CSI for this indication is well-established. As the rate of RCR continues to rise, ${ }^{24}$ it is imperative to better comprehend the implications of postoperative CSI administration to guide clinical decision-making and optimize patient outcomes. The purpose of this study was to review the literature on postoperative CSIs following primary RCR to evaluate efficacy and adverse effects. We hypothesized that postoperative CSIs following primary RCR would be efficacious in improving function and reducing pain, with a low rate of adverse effects.

\section{Methods}

\section{Systematic Review and Study Inclusion}

In November 2019, a systematic review of the MEDLINE, EMBASE, and Cochrane databases was performed according to the Preferred Reporting Item for Systematic Reviews and Meta-Analyses (PRISMA) guidelines $^{25}$ (Fig 1). The following search term was entered into the respective interface, with no date restriction applied: ([rotator cuff OR supraspinatus OR infraspinatus OR subscapularis] AND [injection OR steroid OR corticosteroid OR cortisone OR glucocorticoid OR methylprednisolone OR triamcinolone OR dexamethasone OR betamethasone]). The titles and abstracts of articles identified by this query were then screened by 2 independent reviewers (R.P., B.P.) according to the following inclusion criteria: (1) publication in the English language; (2) publication within the past 15 years; (3) studies reporting on outcomes or adverse effects of CSIs following RCR. Following screening, full-text assessments of all identified articles were performed to confirm inclusion. If there was any ambiguity regarding the potential inclusion of a study based on the title or abstract, a full-text review was conducted. Studies were excluded if they (1) were case reports with 3 or fewer patients, (2) were systematic review articles, (3) reported on revision RCR or concomitant major procedures in addition to RCR, such as fracture fixation, and (4) reported only on preoperative CSIs. Papers listed in the references section of all studies that met the aforementioned inclusion criteria were also screened and evaluated for potential inclusion.

\section{Evaluation of Literature Quality}

Evaluation of all included studies was performed by 2 independent reviewers (B.P. and R.P.), using the Methodological Index for Non-Randomized Studies (MINORS) criteria $^{26}$ or the Cochrane Risk of Bias (CROB) tool ${ }^{27}$ in the case of randomized controlled trials (RCTs). MINORS criteria assess 8 critical aspects of study design for noncomparative clinical studies and an additional 4 aspects of study design for comparative clinical studies. Each item is scored from zero to two- "zero" reflects that the information in question was not reported, whereas "one" reflects that information was reported but was inadequate, and "two" reflects that information was reported and was adequate. Therefore, the maximum possible score is 16 for noncomparative studies and 24 for comparative studies.

The C-ROB tool evaluates RCTs in 5 domains that may predispose to bias: randomization, deviations from intended interventions, missing outcomes data, measurement of the outcome, and selection of the reported result. By answering signaling questions with regard to study methodology, raters grade each domain as having high ROB, some concern for bias, or low ROB. An overall assessment is then made based on the assumption that a given level of ROB for an individual domain implies that the study as a whole has ROB at least this severe. In the event of any discrepancy of MINORS score or C-ROB between the 2 reviewers, the item in question was discussed with the senior author who made the final determination.

\section{Data Collection and Presentation}

For all included papers, information regarding study publication, design, and methodology was extracted. In addition, patient demographics and outcomes data were recorded, including patient-reported outcome measures (PROMs), range of motion (ROM), strength testing, and imaging studies. Finally, all instances of adverse effects or clinical complications were tabulated

\section{Statistical Analysis}

Upon initial review of the included investigations, we found that only one study was a Level of Evidence 
Fig 1. Preferred Reporting Item for Systematic Reviews and Meta-Analyses (PRISMA) flow diagram for study inclusion.
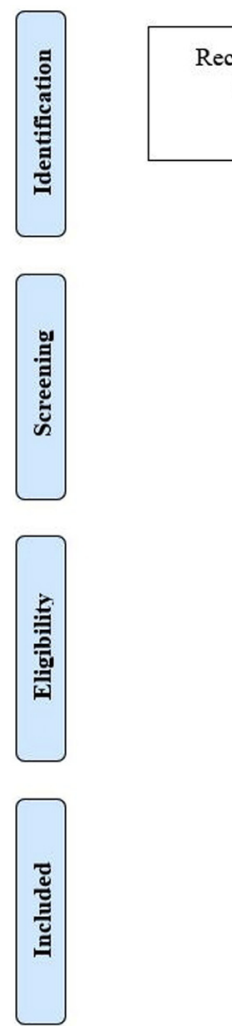

ecords identified through database searching

$(\mathrm{n}=938)$

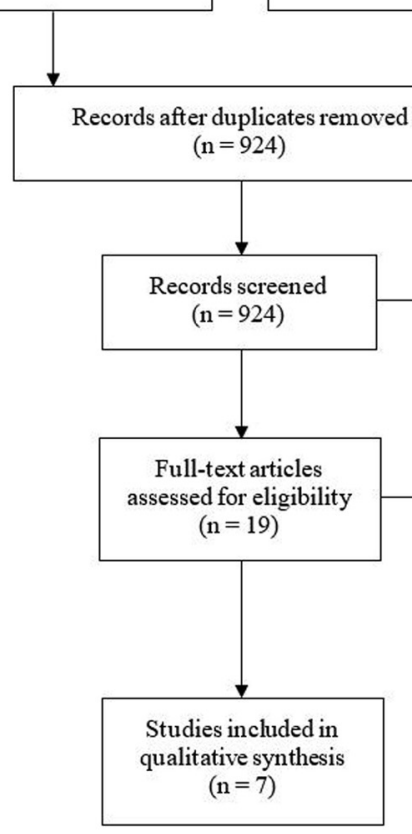

Aditional records identified through other sources $(\mathrm{n}=0)$
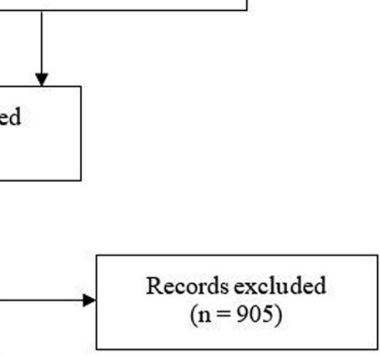

Full-text articles excluded, with reasons $(n=12)$

- Case reports, $\mathrm{n}=2$

- Systematic reviews, $\mathrm{n}=3$

- On preop CSIs only, $\mathrm{n}=7$

(LOE) I RCT, with the remaining studies ranging between LOE IV and LOE II. Therefore, pooling of data was deemed to be inappropriate; instead, data are presented qualitatively by use of tables. Data were tabulated using Excel 365 (Microsoft, Redmond, WA) and evaluated using RStudio software, version 1.0.143 (R Foundation for Statistical Computing, Vienna, Austria).

\section{Results}

\section{Study Characteristics and Patient Demographics}

Our systematic review included 7 unique studies pertaining to corticosteroid injections following RCR $^{9,16-18,20,21,28}$ (Fig 1). Methodologic characteristics of the included papers are summarized in Table 1.9,16-18,20,21,28 In total, 5,528 patients were included, $54.8 \%$ of whom were female, with a mean age range $52.3 \pm 13.0$ to $62.7 \pm 6.6$ years (Table 2 ). None of the included studies reported financial industry support. There was a single Level I prospective RCT, which was determined to have low ROB, whereas the remaining 6 studies were retrospective investigations. None of the included studies reported financial industry support. Among these, the average MINORS score was $20.6 \pm 1.3$ and $13 \pm 1.4$ for comparative $(n=4)$ and noncomparative $(\mathrm{n}=2)$ studies, respectively. In general, studies lost points on MINORS criteria for lack of a priori study size calculations, lack of prospective data collection, and bias related to assessments of end points, although the overall scores were high. In total, our review comprised 5,528 patients, of whom 2,501 were male $(45.2 \%)$. Among the included cohorts, mean age ranged from $52.3 \pm 13.0$ to $62.7 \pm 6.6$ years (Table 2 ).

\section{Efficacy}

Overall, 5 studies reported on the effectiveness of postoperative CSIs. Of these, 4 reported significant improvements in outcome scores among patients who received postoperative CSIs compared with a control group of patients who did receive postoperative CSIs. These relative improvements were found at 3 months postoperatively, but not at later time points (Table 2). The most commonly reported outcome scores among the included studies were the Constant score and the American Shoulder and Elbow Surgeons (ASES) score. Three studies reported on the Constant score-at 3-year follow-up, Baverel et al. ${ }^{9}$ found significantly lower Constant scores in those who received pre- and postoperative CSIs versus those who received neither prenor postoperative injections (Table 3). However, the authors acknowledged that these poorer outcomes in CSI patients were likely the result of pre-existing retears and more reflective of the pathology indicating the CSI rather than a result of the CSIs themselves. Shin et al. ${ }^{20}$ found no difference in Constant score between CSI and 


\begin{tabular}{|c|c|c|c|c|c|c|c|c|}
\hline $\begin{array}{l}\text { First } \\
\text { Author }\end{array}$ & Journal, Year & Region of Origin & Study Design (LOE) & MINORS or C-ROB & $\begin{array}{l}\text { Confounder } \\
\text { Adjustment or Bias } \\
\text { Minimization }\end{array}$ & Patient Source & Inclusion Criteria & $\begin{array}{c}\text { Notable Exclusion } \\
\text { Criteria* }\end{array}$ \\
\hline$\overline{\text { Baverel et al. }{ }^{9}}$ & JSES OA, 2018 & Europe & $\begin{array}{l}\text { Retrospective case } \\
\text { series (IV) }\end{array}$ & $14 / 16$ & $\begin{array}{l}\text { Multivariable } \\
\text { regression }\end{array}$ & Singe institution & $\begin{array}{l}\text { Full thickness RC tear } \\
\text { repaired by double-row } \\
\text { suture technique }\end{array}$ & $\begin{array}{l}\text { - Partial thickness tear } \\
\text { - Hamada stage }>2\end{array}$ \\
\hline Kew et al. ${ }^{16}$ & AJSM, 2019 & North America & $\begin{array}{l}\text { Retrospective cohort } \\
\text { study (III) }\end{array}$ & $20 / 24$ & $\begin{array}{l}\text { Use of } 10: 1 \text { matched } \\
\text { controls; } \\
\text { multivariable } \\
\text { regression }\end{array}$ & $\begin{array}{l}\text { PearlDiver } \\
\text { Database }\end{array}$ & $\begin{array}{l}\text { - Arthroscopic } \\
\text { subacromial } \\
\text { decompression, RC } \\
\text { debridement, or RC } \\
\text { repair }\end{array}$ & $\begin{array}{l}\text { - Previous or } \\
\text { concomitant septic } \\
\text { arthritis }\end{array}$ \\
\hline Kim et al. ${ }^{17}$ & AJSM, 2018 & Asia & $\begin{array}{l}\text { Retrospective cohort } \\
\text { study (III) }\end{array}$ & $19 / 24$ & $\begin{array}{l}\text { Demographically } \\
\text { similar groups }\end{array}$ & Singe institution & $\begin{array}{l}\text { - Repair of full thickness } \\
\text { supraspinatus tear }\end{array}$ & $\begin{array}{l}\text { - Partial-thickness } \\
\text { supraspinatus tear } \\
\text { - } \text { Concomitant biceps } \\
\text { procedure or distal } \\
\text { clavicle excision } \\
\text { - } \text { Preoperative stiffness }\end{array}$ \\
\hline Kim et al. ${ }^{18}$ & AJSM, 2019 & Asia & $\begin{array}{l}\text { Prospective } \\
\text { randomized } \\
\text { controlled trial (I) }\end{array}$ & Low risk of bias & $\begin{array}{l}\text { Double blinded; block } \\
\text { randomization; a } \\
\text { prior sample size } \\
\text { calculation }\end{array}$ & Single institution & $\begin{array}{l}\text { - Repair of small- to } \\
\text { medium-sized RC tear }\end{array}$ & $\begin{array}{l}\text { - } \text { Athletes and heavy } \\
\text { workers } \\
\text { - } \text { RC tear }>2 \mathrm{~cm} \\
\text { - } \text { Preoperative stiffness } \\
\text { or arthritis }\end{array}$ \\
\hline Lee et $\mathrm{al}^{28}$ & KSSTA, 2019 & Asia & $\begin{array}{l}\text { Retrospective case } \\
\text { series (III) }\end{array}$ & $22 / 24$ & $\begin{array}{l}\text { Demographically } \\
\text { similar groups }\end{array}$ & Singe institution & $\begin{array}{l}\text { - Repair of partial RC tear } \\
\text { or small- to medium- } \\
\text { sized full-thickness RC } \\
\text { tear }\end{array}$ & $\begin{array}{l}\text { - } \text { Partial repair } \\
\text { - Worker's } \\
\text { compensation status } \\
\text { - } \text { Repair under tension } \\
\text { requiring } 6 \mathrm{wk} \\
\text { postoperative } \\
\text { immobilization }\end{array}$ \\
\hline Shin et al. ${ }^{20}$ & AJSM, 2016 & Asia & $\begin{array}{l}\text { Retrospective cohort } \\
\text { study (III) }\end{array}$ & $20 / 24$ & $\begin{array}{l}\text { Single blinded; } \\
\text { multivariable } \\
\text { regression }\end{array}$ & Singe institution & $\begin{array}{l}\text { - Repair of partial- } \\
\text { thickness RC tear } \\
>50 \% \text {, or full-thickness } \\
\text { tear }\end{array}$ & $\begin{array}{l}\text { - Worker's } \\
\text { compensation status }\end{array}$ \\
\hline Skedros et al. ${ }^{21}$ & $\begin{array}{l}\text { Pain and } \\
\text { Therapy, } 2017\end{array}$ & North America & $\begin{array}{l}\text { Retrospective case } \\
\text { series (IV) }\end{array}$ & $12 / 16$ & None & Singe institution & $\begin{array}{l}\text { - Nonarthroplasty } \\
\text { shoulder surgery }\end{array}$ & $\begin{array}{l}\text { - Stiffness } 2 / 2 \\
\text { glenohumeral } \\
\text { osteoarthritis } \\
\text { - Postoperative } \\
\text { neurologic } \\
\text { complication, } \\
\text { delayed wound } \\
\text { healing, infection, or } \\
\text { suspicion of infection }\end{array}$ \\
\hline
\end{tabular}


Table 2. Summary of Demographics and Major Findings From All Included Studies

\begin{tabular}{|c|c|c|c|c|c|c|c|c|}
\hline First Author, Cohort & $\mathrm{N}(\mathrm{M}: \mathrm{F})$ & Mean Age, y & Follow-up & $\begin{array}{l}\text { Postoperative CSI } \\
\text { Schedule }\end{array}$ & $\begin{array}{l}\text { Method and } \\
\text { Location of CSI }\end{array}$ & Steroid, Dose & $\begin{array}{l}\text { Significant } \\
\text { Efficacy? }\end{array}$ & $\begin{array}{l}\text { Significant Adverse } \\
\text { Effects? }\end{array}$ \\
\hline \multicolumn{9}{|l|}{ Baverel et al. ${ }^{9}$} \\
\hline $\begin{array}{l}\text { Postoperative CSI } \\
\text { only }\end{array}$ & $31(21: 10)$ & $52.7 \pm 8.0$ & $3.3 \pm 1.2 \mathrm{y}$ & $\begin{array}{l}\text { Mean 1.4 CSIs } \\
\text { (range 1-4), timing }\end{array}$ & & Betamethasone, $5 \mathrm{mg}$ & No & Yes \\
\hline No CSI & $35(21: 14)$ & $52.3 \pm 13.0$ & $3.2 \pm 0.9 y$ & NR & US-guided, subacromial & & & \\
\hline $\begin{array}{l}\text { Preoperative CSI } \\
\text { only }\end{array}$ & $68(41: 27)$ & $58.8 \pm 7.0$ & $3.1 \pm 1.1 \mathrm{y}$ & & & & & \\
\hline $\begin{array}{l}\text { Preoperative and } \\
\text { postoperative } \\
\text { CSI }\end{array}$ & $78(30: 48)$ & $55.4 \pm 10.1$ & $3.0 \pm 0.9 y$ & & & & & \\
\hline \multicolumn{9}{|l|}{ Kew et al. ${ }^{16}$} \\
\hline Humana insured & $1,648(730: 918)$ & NR & Up to 4 mo & 1 CSI within 4 & NR & NR & NR & Yes $^{\dagger}$ \\
\hline Medicare insured & $2,298(964: 1334)$ & NR & & months & & & & \\
\hline \multicolumn{9}{|c|}{ Kim et al. ${ }^{17}$} \\
\hline $\begin{array}{l}\text { Postoperative } \\
\text { CSIs starting at } \\
6 \mathrm{wk}\end{array}$ & $35(12: 23)$ & $58.1 \pm 7.2$ & Min. 2 y & $\begin{array}{l}\text { 1 CSI every } 2 \mathrm{wk} \text { for } \\
6 \mathrm{wk} \text { total, starting } \\
\text { at } 6 \mathrm{wk} \text { vs } 12 \mathrm{wk}\end{array}$ & & Triamcinolone, $20 \mathrm{mg}$ & Yes & No \\
\hline $\begin{array}{l}\text { Postoperative } \\
\text { CSIs starting at } \\
12 \mathrm{wk}\end{array}$ & $39(21: 18)$ & $62.7 \pm 6.6$ & & postop & $\begin{array}{l}\text { US-guided, } \\
\text { glenohumeral }\end{array}$ & & & \\
\hline No CSI & $135(63: 72)$ & $60.0 \pm 7.3$ & & & & & & \\
\hline \multicolumn{9}{|l|}{ Kim et al. ${ }^{18}$} \\
\hline Postoperative SI & $40(17: 23)$ & $59.8 \pm 8.4$ & $23.1 \pm 1.8 \mathrm{mo}$ & 1 CSI at 8 weeks & $\begin{array}{l}\text { US-guided, } \\
\text { glenohumeral }\end{array}$ & Triamcinolone, $40 \mathrm{mg}$ & Yes & No \\
\hline No CSI & $40(21: 19)$ & $60.4 \pm 8.6$ & $27.0 \pm 2.3 \mathrm{mo}$ & & & & & \\
\hline \multicolumn{9}{|l|}{ Lee et $a^{28}$} \\
\hline Postoperative SI & $56(24: 32)$ & $60.9 \pm 7.3$ & Min. 2 y & $1 \mathrm{CSI}$ at 3 months & $\begin{array}{l}\text { US-guided, } \\
\text { glenohumeral }\end{array}$ & Triamcinolone, 20mg & Yes & No \\
\hline No CSI & $262(106: 156)$ & $61.3 \pm 7.8$ & & & & & & \\
\hline \multicolumn{9}{|l|}{ Shin et al. ${ }^{20}$} \\
\hline Postoperative SI & $72(38: 34)$ & $57.3 \pm 8.6$ & Min. 2 y & 1 CSI at mean & US-guided, subacromial & Triamcinolone, 40mg & Yes & No \\
\hline No CSI & $386(203: 183)$ & $57.3 \pm 8.5$ & & $34 \pm 5$ days & & & & \\
\hline \multicolumn{9}{|l|}{ Skedros et al. ${ }^{21}$} \\
\hline Postoperative CSI & $58(32: 26)$ & $53 \pm 13$ & Min. $12 \mathrm{wk}$ & 1 CSI at mean & & Methylprednisolone, 80 & NR & No \\
\hline No CSI & $247(157: 90)$ & $58 \pm 12$ & & $88 \pm 38$ days & $\begin{array}{l}\text { No imaging guidance, } \\
\text { subacromial or } \\
\text { glenohumeral }\end{array}$ & $\begin{array}{c}\text { mg or } 160 \mathrm{mg} \text { at provider } \\
\text { discretion }\end{array}$ & & \\
\hline
\end{tabular}

CSI, corticosteroid injection; F, female; M, male; NR, not reported, SI, steroid injection; US, ultrasound.

*Results for these parameters are based on the authors' primary findings and their resultant recommendations for postoperative CSI use.

${ }^{\dagger}$ Kew et al. found a significant increase in infection risk only if CSI was administered within 1 month following surgery; CSIs administered at later intervals did not confer increased risk. 


\section{averel et al.}

Constant: $54.7 \pm 17.5$

Constant: $58.4 \pm 12.6$

C. CSI preoperative only, $\mathrm{n}=68$

Constant: $55.6 \pm 15.0$

D. CSI pre- and postoperative, $\mathrm{n}=78$ Kim et al. ${ }^{17}$

A. CSIs starting at $6 \mathrm{wk}, \mathrm{n}=35$

Constant: $56.8+15.3$

KSS: $61.2 \pm 11.7$

UCLA: $22.0 \pm 4.2$

KSS: $58.5 \pm 17.0$

B. CSIs starting at $12 \mathrm{wk}, \mathrm{n}=39$

C. No CSI, $\mathrm{n}=135$

KSS: $66.3 \pm 15.0$
+25.2 (preoperative to $3 \mathrm{y}, \mathrm{NR}$ )

+28.6 (preoperative to $3 \mathrm{y}, \mathrm{NR}$ )

+28.5 (preoperative to $3 \mathrm{y}, \mathrm{NR}$ )

+18.6 (preoperative to $3 \mathrm{y}, \mathrm{NR}$ )

+20.1 (preoperative to $2 \mathrm{y}, P<.001$ )

+7.3 (preoperative to $2 \mathrm{y}, P<.001$ )

+24.9 (preoperative to $2 \mathrm{y}, P<.001$ )

+9.4 (preoperative to $2 \mathrm{y}, P<.001$ )

+24.1 (preoperative to $2 \mathrm{y}, P<.001$ )

UCLA: $22.0 \pm 4.8$
Kim et al. ${ }^{18}$

A. CSI, $\mathrm{n}=40$

B. No CSI, $\mathrm{n}=40$
ASES: $66.4 \pm 17.2$

VAS: 4

Constant: 60.3

ASES: $62.1 \pm 18.5$

VAS: 4.7

Constant: 62.8
+24.7 (preoperative to $6 \mathrm{mo}, P=.01$ )

-2.9 (preoperative to $6 \mathrm{mo}, P=.03$ )

+17.5 (preoperative to $6 \mathrm{mo}, P=.02$ )

+27.7 (preoperative to $6 \mathrm{mo}, P=.03$ )

-3.5 (preoperative to $6 \mathrm{mo}, P=.02$ )

+15.4 (preoperative to $6 \mathrm{mo}, P=.02$ )

\section{B vs D}

Constant at 3 y: $<.05$

Other groups not significantly different from each other in Constant score at $3 \mathrm{y}$.

$$
\text { A vs } B
$$

KSS at 3 mo: $<.001$

KSS at 6 mo, $1 \mathrm{y}, 2 \mathrm{y}:>.05$

UCLA at 3 mo: $<.001$

UCLA at 6 mo, 1 y, 2 y: $>.05$

$$
\text { A vs } C
$$

KSS at 3 mo: .990

KSS at 6 mo: .060

KSS at 1 y, 2 y: $<.05$

UCLA at $3 \mathrm{mo}, 6 \mathrm{mo}, 1 \mathrm{y}:>.05$

UCLA at $2 \mathrm{y}:<.05$

$$
\mathrm{B} \text { vs } \mathrm{C}
$$

KSS at $3 \mathrm{mo}, 6 \mathrm{mo}, 1 \mathrm{y}, 2 \mathrm{y}:<.05$

UCLA at $3 \mathrm{mo}, 6 \mathrm{mo}, 2 \mathrm{Y}:<.05$

UCLA at $1 \mathrm{y}:>.05$

A vs B

ASES preoperative: 0.27

ASES at 3 mo: .02

ASES at 6 mo: $P>.05$

VAS preoperative: NR

VAS at 3 mo: .02

VAS at $6 \mathrm{mo}:>.05$ 


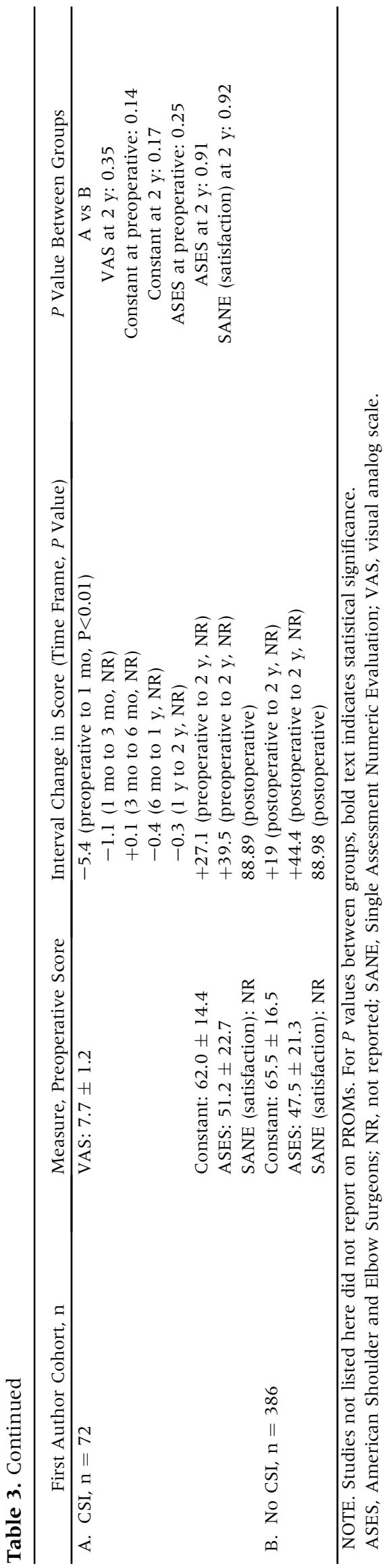

non-CSI patients at 2-year follow-up, whereas Kim et al. ${ }^{29}$ found similar Constant scores between CSI and non-CSI patients at 6-month follow-up, though comparative statistics were not reported. Three studies reported on ASES scores, 2 of which reported significantly greater improvements among CSI patients versus non-CSI patients at 3 months postoperatively. However, in both cases, these improvements were not statistically greater than the non-CSI group at later follow-up time points ${ }^{18,28}$ (Table 3). In addition, the studies by Shin et al., ${ }^{20}$ Kim et al., ${ }^{18}$ and Lee et al. ${ }^{28}$ reported significant reductions in pain at 1 month, 3 months, and 3 months post-CSI, respectively. Other PROMs were reported by fewer than 3 studies, and complete reporting of PROMs from all included investigations are displayed in Table 3.

ROM data were reported by 3 included investigations. Kim et al. ${ }^{17}$ found that ROM was improved in patients who received CSIs starting at 6 weeks postoperatively when compared with those starting injections at 12 weeks postoperatively. Lee et al. ${ }^{28}$ found significantly better ROM in forward flexion in those who received CSIs versus those who did not at 3 months' postoperatively. There were no significant differences between groups in forward flexion at 6-, 12-, or 24month follow-up, nor were there any significant differences in external rotation or internal rotation at any time point. Finally, Baverel et al. ${ }^{9}$ reported significantly better ROM in patients who received no CSIs, when compared with those who received only preoperative injections, those who received only postoperative injections, and those who received both pre- and postoperative injections $(P=.001)$.

\section{Adverse Effects}

Several included investigations reported on adverse effects of postoperative CSIs. Kew et al. ${ }^{16}$ found that among 3,946 patients, 264 received a CSI within 1 month following arthroscopic shoulder surgery, and these patients had a significantly increased risk of infection (privately insured; odds ratio [OR] 2.63; 95\% confidence interval $[\mathrm{CI}]$ 1.32-5.22, $P=.014$ ) (Medicare insurance; OR 11.2; 95\% CI 2.33-53.57, $P<.0001)$. Conversely, injections from 2 to 4 months postoperatively were not significantly associated with a greater risk for infection in either cohort. However, the authors included multiple types of arthroscopic shoulder surgery in their investigation, including subacromial Decompression, rotator cuff debridement, lysis of adhesions, synovectomy, and RCR, and did not stratify their results by procedure. The study by Kim et al. ${ }^{17}$ reported that retear rates were $5.7 \%, 10.8 \%$, and $14.1 \%$ at minimum 2-year follow-up for patients who started CSIs at 6 weeks postoperatively, 12 weeks postoperatively, and those who did not receive CSIs, respectively $(P=.374)$. All patients included in this 
study had received a postoperative magnetic resonance imaging (MRI) scan at 6 months, with retears defined as Sugaya type IV or $\mathrm{V} .{ }^{17}$ Lee et al. ${ }^{28}$ reported no significant difference in retear rate between CSI and nonCSI patients, as evaluated by magnetic resonance arthrography at 6 months postoperatively (CSI: $17.9 \%$ vs no CSI: $17.2 \%, P>.05)$. All patients included in this study received the postoperative imaging, and retear was defined as a stage 3 or 4 tear according to the French Society of Arthroscopy. ${ }^{28}$ Shin et al. ${ }^{20}$ reported similar results, also at 6 months postoperatively, based on MRI studies (CSI: $6.8 \%$ vs no CSI: $18.4 \%, P=.06$ ). In this study, $71.2 \%$ of patients received a postoperative MRI at 6 months $(61 \%$ who had received a CSI, $73 \%$ without an injection), and the criteria for retear was not defined. $^{20}$ In contrast to these findings, however, Baverel et al. ${ }^{9}$ found a 2 -fold increase in retear rate, as defined by a Sugaya type IV or V tear on ultrasound evaluation performed on all patients, per each postoperative CSI (OR 2.19; 95\% CI 1.23-2.92, $P=.007$ ). However, the authors of this study were unable to determine whether these retears were present before receiving the postoperative CSI. Finally, Skedros et al. ${ }^{21}$ reviewed 754 patients who underwent nonarthroplasty shoulder surgery, including 305 who underwent RCR. Of these, 58 patients $(19 \%)$ received a postoperative CSI for indications of pain, stiffness, or inflammation/ bursitis within the first 3 postoperative months. There were no minor or major complications in any patients, including no instances of tendon rupture. However, only instances of infections, poor wound healing, dermatitis, and apparent structural compromise on examination, were recorded as complications. A summary of the reported adverse effects is displayed in Table 4.

\section{Discussion}

Studies included in this review reported statistically significant improvements in at least one outcome measure at 3 months postoperatively (Constant, ASES, and visual analog scale score for pain) in patients who received postoperative CSIs when compared with those who did not. However, this significant relative improvement did not persist at 6 months, 1 year, or 2 years, postoperatively. The only study not to find some level of efficacy of postoperative CSIs was conducted by Baverel et al., ${ }^{9}$ and the authors concede that the poor results in CSI patients was more likely a consequence of the degree of rotator cuff retears in this cohort, rather than the CSI itself. In addition, this study was 1 of only 2 investigations to describe a significantly increased risk of adverse effects in the CSI group. In the other case, Kew et al. ${ }^{16}$ found an increased risk of infection only if the CSI was administered within 1 month postoperatively (OR 2.6 and 11.2, for privately insured and Medicare patients, respectively). On the basis of these cumulative results, this systematic review suggests that, if administered at least 1 month after surgery, postoperative CSIs may provide patients with meaningful improvements in function and pain control in the first few months following primary RCR, with a low risk of adverse effects.

The molecular effects of corticosteroids on the inflammatory cascade, healing, and matrix remodeling of tendon continues to be a topic of interest in the orthopaedic literature. Surgical intervention for rotator cuff-related pain should ideally strike a delicate balance between achieving tensile strength for early motion to prevent adhesive scars and catalyze a local inflammatory cascade to promote healing and regeneration of tissue. ${ }^{30}$ Rotator cuff pathology is associated with an abundance of molecular markers, including matrix proteins, growth factors, enzymes, and local inflammatory cells. ${ }^{31}$ Steroids have been shown to decrease collagen matrix remodeling, ${ }^{32,33}$ halt the initial proliferative healing response, ${ }^{15,34}$ prevent tenocyte differentiation, ${ }^{35}$ and reduce tendon biomechanical strength. ${ }^{33,36}$ Still, other basic science studies report that corticosteroids both dampen the initial inflammatory response by increasing nuclear translocation of inhibitory transcription factors and prolong it by decreasing the number of local cytotoxic cells needed to halt inflammation. ${ }^{31,34,36}$ Although current data remain ambiguous, CSIs appear to decrease local inflammation at best and at worst have the potential to interfere with the course of crucial postoperative healing.

The timing of postoperative CSIs appears to be the critical factor in efficacy and safety. Notably, an investigation by Lee et al. ${ }^{32}$ has reported that the detrimental molecular effects of CSIs on collagen composition, extracellular matrix organization, and early healing likely normalize within 6 weeks of administration. This is in line with the aforementioned results of Kew et al., ${ }^{16}$ which indicate that CSIs increase rates of retear if administered within one month post-operatively but not if administered after that time point. In addition, the study by Kim et al. ${ }^{17}$ included in the present review reported persistently improved UCLA Shoulder Scores and Korean Shoulder Scores for up to 2 years postoperatively in patients receiving CSIs starting at 12 weeks post-operatively, as compared to those receiving CSIs starting at 6 weeks postoperatively. This finding further supports the concept of the detrimental effect of CSIs only in the very early postoperative period. The remainder of the data accumulated in the present review suggest that at long-term follow-up, patients who receive CSIs outside of this early postoperative window of 4 to 6 weeks achieve a similar level of function as their counterparts who do not receive injections. Taken together, these findings may suggest that any adverse molecular effects of postoperative CSIs on the biomechanical integrity are transient. 
Table 4. Summary of Reported Adverse Effects and Complications of Postoperative CSIs

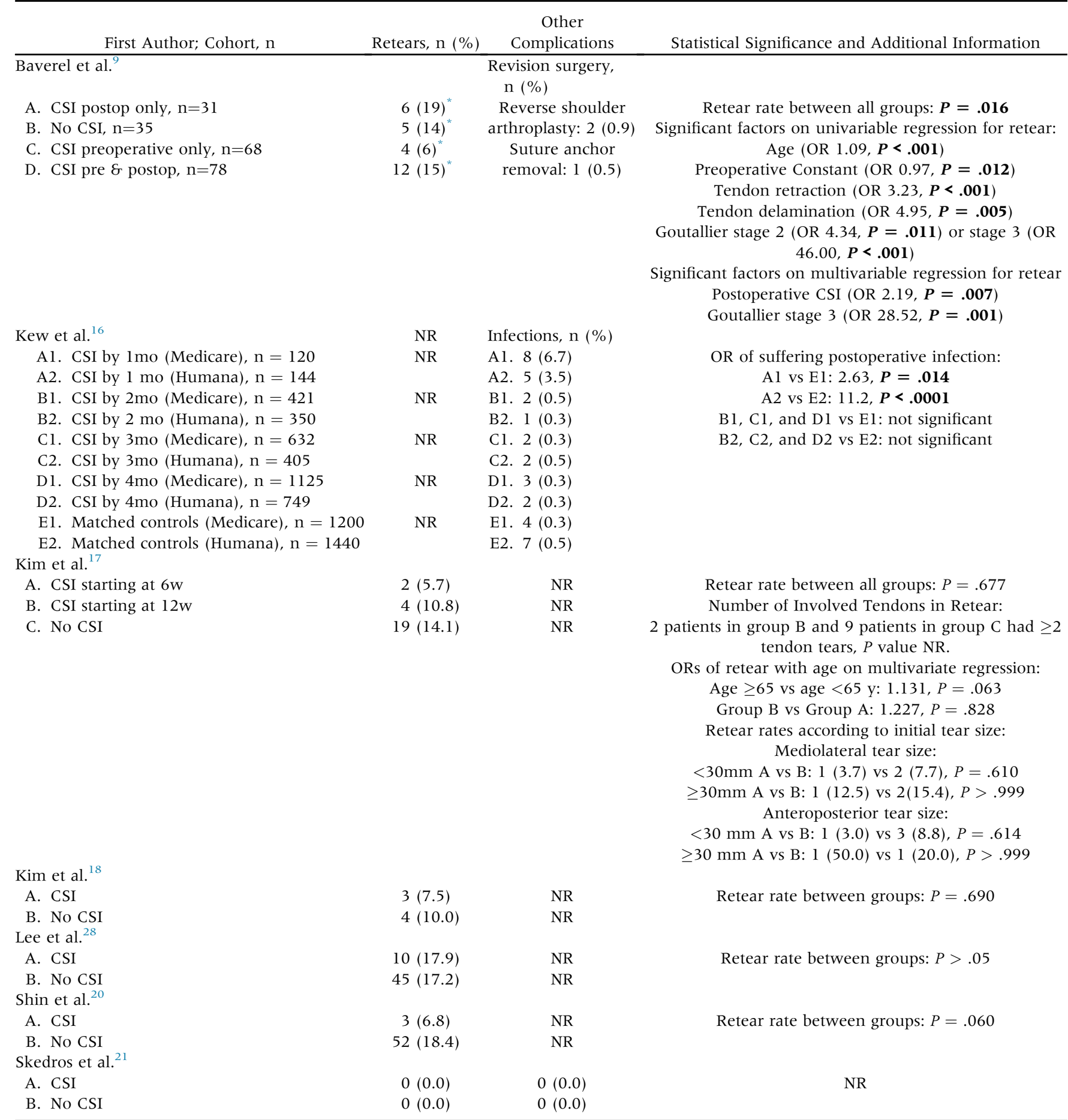

NOTE. $P$ values in bold indicate statistical significance.

CSI, corticosteroid injection; NR, not reported; OR, odds ratio.

*Retear defined as Sugaya Grade IV or V on ultrasound.

The effect of CSIs in the preoperative setting has also been a focal point of the literature in the past several years. In a recent review, Puzzitiello et al. ${ }^{37}$ considered 8 investigations on this topic and found that a single CSI for rotator cuff tendinosis was associated with an increased risk of revision RCR (OR range 1.3 [1.1-1.7] to $2.8[2.2-3.4]$ ), and postoperative infections (OR 2.1 [1.5-2.7]) when administered within a month before RCR. The authors ultimately concluded that a temporal and dose-dependent relationship exists between administration of preoperative CSIs and adverse postoperative effects. Risk mitigation of modifiable 
perioperative factors, including potential postponement of surgery for patients with recent or frequent shoulder injections, have been proposed as a possible approach to minimize CSI complication risk. ${ }^{38}$ However, mitigation of complications is but one factor in the clinical context of CSI after rotator cuff surgery, and CSI's potential for interference with postsurgical healing is critical to thoughtfully consider in clinical decisionmaking.

If deemed necessary in the very early postoperative period, it may be prudent to offer patients struggling with pain and stiffness an aggressive regimen of physical therapy and medical pain control before considering CSIs. The most recent review of the literature on CSI analgesic effects in rotator cuff-related shoulder pain found moderate evidence favoring CSI over anesthetic-only block within the first 2 postoperative months, and strong evidence for no difference between injection composition after 12 weeks postoperatively. ${ }^{39}$ Further studies investigating combination therapy addressing both pain and function, such as one currently enrolling multicenter RCT in the United Kingdom investigating physiotherapy in addition to CSI, are promising. ${ }^{40}$ At present, CSIs appear to be a safe and efficacious management option for improving function and reducing pain if administered after the first postoperative month.

\section{Limitations}

The results of this review should be considered in the context of several limitations. Although selection criteria of the included studies were strict, there was only one investigation with Level I evidence, whereas the others were lesser in quality. This limitation precluded the pooling of data for more involved statistical analysis. Moreover, many of the included nonrandomized studies administered CSIs to patients with persistent postoperative pain or stiffness, whereas the control groups had lesser degree of symptoms and thus were not indicated for a postoperative CSI. However, the efficacy of the postoperative CSI was measured in terms of improvement in outcomes. In addition, the inclusion of several retrospective cohort studies from single institutions may limit the applicability of our results to a broader patient population. Moreover, the included studies were heterogeneous with regard the particular steroid used in the CSI, as well as with regard to dosage and the location of the injection (subacromial injection vs intra-articular injection). Finally, methods of evaluating patients varied among the included investigations, limiting our ability to make comparisons across studies.

\section{Conclusions}

Postoperative CSIs may improve pain and function for up to 3 months following primary RCR but not at later follow-up time points. CSIs should be administered only after the first postoperative month to minimize the potential risk for adverse events.

\section{References}

1. Martin SD, Conaway WK, Lei P. Use of intra-articular corticosteroids in orthopaedics. J Bone Joint Surg Am 2018;100:885-891.

2. Kitridis D, Tsikopoulos K, Bisbinas I, Papaioannidou P, Givissis P. Efficacy of pharmacological therapies for adhesive capsulitis of the shoulder: A systematic review and network meta-analysis. Am J Sports Med 2019;47: 3352-3560.

3. Mohamadi A, Chan JJ, Claessen FM, Ring D, Chen NC. Corticosteroid injections give small and transient pain relief in rotator cuff tendinosis: A meta-analysis. Clin Orthop Relat Res 2017;475:232-243.

4. Park HB, Kam M, Gwark JY. Association of steroid injection with soft-tissue calcification in lateral epicondylitis. J Shoulder Elbow Surg 2019;28:304-309.

5. Evans CH, Kraus VB, Setton LA. Progress in intra-articular therapy. Nat Rev Rheumatol 2014;10:11-22.

6. MacMahon PJ, Eustace SJ, Kavanagh EC. Injectable corticosteroid and local anesthetic preparations: A review for radiologists. Radiology 2009;252:647-661.

7. Ranalletta M, Rossi LA, Bongiovanni SL, Tanoira I, Elizondo CM, Maignon GD. Corticosteroid injections accelerate pain relief and recovery of function compared with oral NSAIDs in patients with adhesive capsulitis: A randomized controlled trial. Am J Sports Med 2016;44: 474-481.

8. Lin MT, Chiang CF, Wu CH, Huang YT, Tu YK, Wang TG. Comparative effectiveness of injection therapies in rotator cuff tendinopathy: A systematic review, pairwise and network meta-analysis of randomized controlled trials. Arch Phys Med Rehabil 2019;100:336-349.e315.

9. Baverel L, Boutsiadis A, Reynolds RJ, Saffarini M, Barthelemy R, Barth J. Do corticosteroid injections compromise rotator cuff tendon healing after arthroscopic repair? JSES Open Access 2018;2:54-59.

10. Weber AE, Trasolini NA, Mayer EN, et al. Injections prior to rotator cuff repair are associated with increased rotator cuff revision rates. Arthroscopy 2019;35:717-724.

11. Agarwalla A, Puzzitiello RN, Mascarenhas R, Sumner S, Romeo AA, Forsythe B. Preoperative injections may be an iatrogenic cause of reoperation after arthroscopic rotator cuff repair. Arthroscopy 2019;35:325-331.

12. Forsythe B, Agarwalla A, Puzzitiello RN, Sumner S, Romeo AA, Mascarenhas R. The timing of injections prior to arthroscopic rotator cuff repair impacts the risk of surgical site infection. J Bone Joint Surg Am 2019;101: 682-687.

13. Desai VS, Camp CL, Boddapati V, Dines JS, Brockmeier SF, Werner BC. Increasing numbers of shoulder corticosteroid injections within a year preoperatively may be associated with a higher rate of subsequent revision rotator cuff surgery. Arthroscopy 2019;35:45-50.

14. Traven SA, Brinton D, Simpson KN, et al. Preoperative shoulder injections are associated with increased risk of revision rotator cuff repair. Arthroscopy 2019;35:706-713. 
15. Dean BJ, Franklin SL, Murphy RJ, Javaid MK, Carr AJ. Glucocorticoids induce specific ion-channel-mediated toxicity in human rotator cuff tendon: a mechanism underpinning the ultimately deleterious effect of steroid injection in tendinopathy? Br J Sports Med 2014;48: 1620-1626.

16. Kew ME, Cancienne JM, Christensen JE, Werner BC. The timing of corticosteroid injections after arthroscopic shoulder procedures affects postoperative infection risk. Am J Sports Med 2019;47:915-921.

17. Kim IB, Jung DW. An intra-articular steroid injection at 6 weeks postoperatively for shoulder stiffness after arthroscopic rotator cuff repair does not affect repair integrity. Am J Sports Med 2018;46:2192-2202.

18. Kim YS, Jin HK, Lee HJ, Cho HL, Lee WS, Jang HJ. Is it safe to inject corticosteroids into the glenohumeral joint after arthroscopic rotator cuff repair? Am J Sports Med 2019;47:1694-1700.

19. Perdreau A, Joudet T. Efficacy of multimodal analgesia injection combined with corticosteroids after arthroscopic rotator cuff repair. Orthop Traumatol Surg Res 2015;101: S337-345.

20. Shin SJ, Do NH, Lee J, Ko YW. Efficacy of a subacromial corticosteroid injection for persistent pain after arthroscopic rotator cuff repair. Am J Sports Med 2016;44: 2231-2236.

21. Skedros JG, Adondakis MG, Knight AN, Pilkington MB. Frequency of shoulder corticosteroid injections for pain and stiffness after shoulder surgery and their potential to enhance outcomes with physiotherapy: A retrospective study. Pain Ther 2017;6:45-60.

22. Huberty DP, Schoolfield JD, Brady PC, Vadala AP, Arrigoni P, Burkhart SS. Incidence and treatment of postoperative stiffness following arthroscopic rotator cuff repair. Arthroscopy 2009;25:880-890.

23. Chung SW, Huong CB, Kim SH, Oh JH. Shoulder stiffness after rotator cuff repair: Risk factors and influence on outcome. Arthroscopy 2013;29:290-300.

24. Austin DC, Torchia MT, Lurie JD, Jevsevar DS, Bell JE. Identifying regional characteristics influencing variation in the utilization of rotator cuff repair in the United States. J Shoulder Elbow Surg 2019;28:1568-1577.

25. Moher D, Liberati A, Tetzlaff J, Altman DG. Preferred reporting items for systematic reviews and meta-analyses: The PRISMA statement. Ann Intern Med 2009;151:264-269.

26. Slim K, Nini E, Forestier D, Kwiatkowski F, Panis Y, Chipponi J. Methodological index for non-randomized studies (minors): Development and validation of a new instrument. ANZ J Surg 2003;73:712-716.

27. Higgins J, Sterne J, Savovic J, et al. A revised tool for assessing risk of bias in randomized trials. Cochrane Database Systematic Rev 2016;10:29-31 (suppl 1).
28. Lee W, Kim SJ, Choi CH, Choi YR, Chun YM. Intraarticular injection of steroids in the early postoperative period does not have an adverse effect on the clinical outcomes and the re-tear rate after arthroscopic rotator cuff repair. Knee Surg Sports Traumatol Arthrosc 2019;27: 3912-3919.

29. Kim YS, Lee HJ, Lee DH, Choi KY. Comparison of highand low-dose intra-articular triamcinolone acetonide injection for treatment of primary shoulder stiffness: A prospective randomized trial. J Shoulder Elbow Surg 2017;26:209-215.

30. Myer C, Fowler JR. Flexor tendon repair: Healing, biomechanics, and suture configurations. Orthop Clin North Am 2016:47:219-226.

31. Dean BJ, Franklin SL, Carr AJ. A systematic review of the histological and molecular changes in rotator cuff disease. Bone Joint Res 2012;1:158-166.

32. Lee HJ, Kim YS, Ok JH, Lee YK, Ha MY. Effect of a single subacromial prednisolone injection in acute rotator cuff tears in a rat model. Knee Surg Sports Traumatol Arthrosc 2015;23:555-561.

33. Riley G. The pathogenesis of tendinopathy. A molecular perspective. Rheumatology (Oxford) 2004;43:131-142.

34. Ji JH, Kim YY, Patel K, et al. Dexamethasone facilitates NF-kappaB signal pathway in TNF-alpha stimulated rotator cuff tenocytes. J Microbiol Biotechnol 2019;29: 297-303.

35. Chen W, Tang $\mathrm{H}$, Zhou $\mathrm{M}$, Hu C, Zhang J, Tang K. Dexamethasone inhibits the differentiation of rat tendon stem cells into tenocytes by targeting the scleraxis gene. J Steroid Biochem Mol Biol 2015;152:16-24.

36. Blomgran P, Hammerman M, Aspenberg P. Systemic corticosteroids improve tendon healing when given after the early inflammatory phase. Sci Rep 2017;7:12468.

37. Puzzitiello RN, Patel BH, Nwachukwu BU, Allen AA, Forsythe B, Salzler MJ. Adverse impact of corticosteroid injection on rotator cuff tendon health and repair: A systematic review. Arthroscopy 2020;36:1468-1475.

38. Lubowitz JH, Brand JC, Rossi MJ. Preoperative shoulder corticosteroid injection is associated with revision after primary rotator cuff repair. Arthroscopy 2019;35:693-694.

39. Cook T, Minns Lowe C, Maybury M, Lewis JS. Are corticosteroid injections more beneficial than anaesthetic injections alone in the management of rotator cuff-related shoulder pain? A systematic review. Br J Sports Med 2018;52:497-504.

40. Hopewell S, Keene DJ, Maia Schlussel M, et al. Clinical and cost-effectiveness of progressive exercise compared with best practice advice, with or without corticosteroid injection, for the treatment of rotator cuff disorders: Protocol for a 2x2 factorial randomised controlled trial (the GRASP trial). BMJ Open 2017;7:e018004. 